\title{
HYDROGEN DYNAMICS IN MAGNESIUM AND GRAPHITE
}

\author{
N. Jacobson, B. Tegner, E. Schröder, P. Hyldgaard ${ }^{1}$, and B. I. Lundqvist \\ Department of Applied Physics, Chalmers University of Technology and Göteborg University, \\ S-412 96 Göteborg, Sweden
}

\begin{abstract}
Magnesium, nanotubes, graphitic nanofibers, and graphite represent interesting opportunities for efficient hydrogen storage applications and motivate first-principle investigations of the hydrogen energetics and dynamics that describe such potential technological usage. We present spin-polarized electron-density calculations of adsorption energies and diffusion barriers for both chemisorbed and physisorbed hydrogen atoms to provide a detailed description of the important hydrogen dynamics.
\end{abstract}

Keywords: Hydrogen storage, graphite and magnesium hydrogen dynamics, first-principle calculations.

\footnotetext{
${ }^{1}$ Corresponding author: Tel. +46 31772 8422; Fax: +46 31772 8426; E-mail: hyldgaar@fy.chalmers.se
} 


\section{Introduction}

Hydrogen is a strong alternative to fossil fuels and could be used in both vehicles and for domestic heating. It can be made available in huge quantities via electrolysis of water and represents an efficient, clean, and environmentally friendly energy carrier which, upon combustion, produces nothing but water. The hydrogen energy content, $124 \mathrm{MJ} / \mathrm{kg}$, is much higher than other chemical fuels like petroleum (at $44 \mathrm{MJ} / \mathrm{kg}$ ). The significant hydrogen chemical energy can be used to run both combustion engines and fuelcells. It is, however, necessary to develop efficient and safe hydrogen storage. For vehicular use, in particular, it is important that the storage device is also (crash-safe and) sufficiently light to make the hydrogen fuel cost effective. The U.S Department of Energy has set a minimum storage goal of $6.5 \%$ hydrogen by weight for commercial applications [1]. Traditional pressure tanks are much too heavy and there is a significant interest in using, for example, graphitic materials (graphite, nanotubes and nanofibers) [2-9] for hydrogen storage.

Here we use density functional theory (DFT) [10, 11] to study both hydrogen storage and hydrogen uptake in the two common, non-toxic, and accessible materials: magnesium [12] and graphite. In magnesium the hydrogen gains $0.5 \mathrm{eV}$ per atom when forming a magnesium hydride $\mathrm{MgH}_{2}$ (7.7\% hydrogen by weight). Graphite, we find, could store hydrogen at $0.06 \mathrm{eV}$ per molecule through swelling (intercalation) with one $\mathrm{H}_{2}$ molecule per two carbon atoms $(7.7 \%$ hydrogen by weight). However, while pure graphite is described by the interlayer separation

$c_{0}=3.35 \AA$, such $\mathrm{H}_{2}$ intercalation causes a dramatic interlayer relaxation, $c_{0} \rightarrow c \approx 1.70 c_{0}$. For both of the storage materials we find that the hydrogen dynamics could limit and/or control the actual hydrogen uptake and release.

This paper is organized as follows. Section 2 provides an overview of our investigations, while sections 3 and 4 present details of our calculations of key hydrogen-dynamics dynamics effects within magnesium and graphite. 


\section{First-principle study of hydrogen-storage dynamics}

We seek a refined discussion of the magnesium and graphite potential as hydrogen storage materials. Calculations of the energy gain (summarized above) by hydrogen when forming a hydride or an intercalation state, can determine the equilibrium statistical mechanical properties but actual storage operation, actual hydrogen uptake and release may be inhibited by dynamical effects and/or significant bottlenecks in the form of large energy barriers for hydrogen entry/release.

We investigate such hydrogen-dynamics effects for both of the storage materials in DFT using both the local density approximation (LDA) and the generalized gradient approximation (GGA) in a plane-wave code and (when necessary) using a spin-polarized description. For investigations of hydrogen surface dynamics we include a slice of vacuum above the surface. Details are given in the relevant sections. For the magnesium case we focus on the atomic hydrogen diffusion and report binding energies per atom with respect to the energy, $E_{\mathrm{H}_{2}} / 2+E_{\mathrm{Mg}}$, of one half an undissociated $\mathrm{H}_{2}$ molecule plus a clean magnesium system. For graphite hydrogen storage the

emphasis is on molecular hydrogen uptake and here we report binding energies per molecule relative to the energy $E_{\mathrm{H}_{2}}+E_{\mathrm{G}}$ of the molecule plus a clean graphite system. Through such accurate such first-principle calculations we determine both the hydrogen-storage energy gain itself as well as important hydrogen-dynamics effects.

\section{Hydrogen storage in magnesium}

The upper right panel of Fig. 1 shows a schematic of the $\operatorname{Mg}(0001)$ surface which has a sixfold symmetry with hollow 'shafts' (perpendicularly to the surface) which extend into the Mg bulk. The hcp positions (A and B) and fcc positions (C) are shown with Mg atoms in the A sites comprising the top layer. Deeper layers are hidden by the two top layers. 

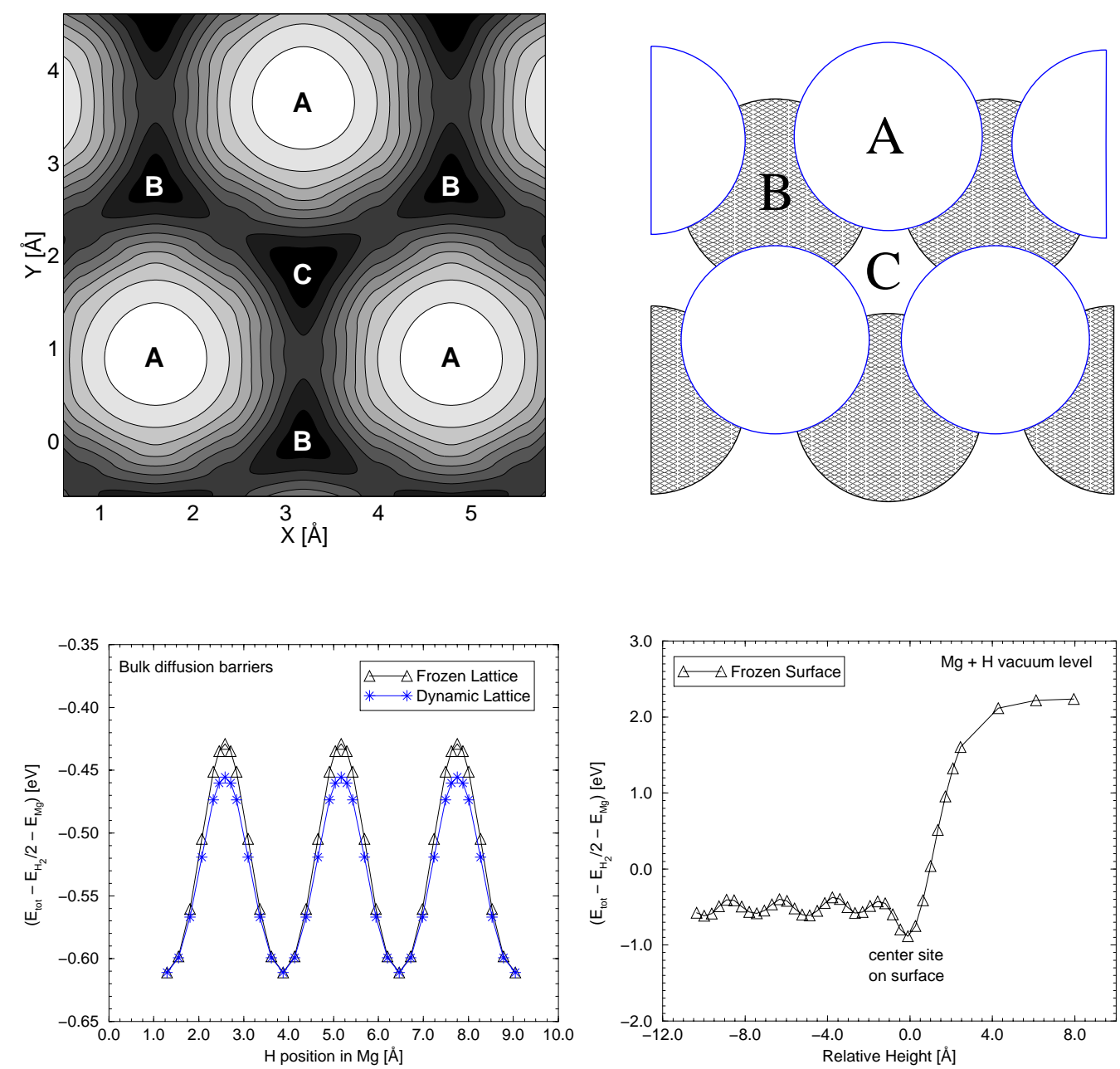

Figure 1: Hydrogen dynamics on and in magnesium. The upper right panel sketches the clean $\mathrm{Mg}(0001)$ surface seen from above and defines a set of possible high-symmetry positions for the atomic hydrogen: top sites (A), hcp sites (B), and fcc sites (C). The upper left panel shows a calculated contour plot of the binding energy of a single $\mathrm{H}$ atom on top of a (frozen) $\operatorname{Mg}(0001)$ surface. The lower right panel shows the calculated potential energy curve for moving a hydrogen atom from the $\mathrm{Mg}(0001)$ surface and down along a set of fcc C-sites. Finally, the lower left panel shows the diffusion barriers for atomic hydrogen deep in the $\mathrm{Mg}$ bulk moving along the shaft of C-sites. 
The upper left panel of Fig. 1 shows a contour plot of the potential-energy surface (PES) for atomic hydrogen on the frozen $\mathrm{Mg}(0001)$ surface. The minimum energy value is the fcc site $\mathrm{C}$ at $-0.88 \mathrm{eV}$ and the maximum value is the top site $\mathrm{A}$ at $-0.09 \mathrm{eV}$. The contour step is $0.08 \mathrm{eV}$. We calculate this PES within DFT using GGA and a $2 \times 3 \times 6$ supercell with the equivalent of 8 vacuum layers above the $\mathrm{Mg}$ slab. In all cases the magnesium bond lengths were fixed after the $\mathrm{H}$ atom was introduced into the system. Hydrogen dynamics was restricted to the vertical direction, due to the unstable sites on the surface. We find that a single hydrogen atom can adsorb at B and C-sites consistent with experimental work reported by Sprunger and Plummer [13]. We find a preference for adsorption at C-sites with a binding-energy difference $E_{B}-E_{C}=28 \mathrm{meV}$ consistent with previous theoretical work [14].

The lower right panel of Fig. 1 shows the calculated potential energy curve for atomic hydrogen taken through the $\mathrm{Mg}(0001)$ surface and into the bulk via the fcc Mg 'shaft' (C-sites). With the surface fcc sites being the preferred position for atomic H, this diffusion represents the expected path for hydrogen penetration and magnesium hydride formation. The bond lengths of the Mg-atoms where kept fixed for this calculation. We predict, as shown in the panel, an entry barrier of around $500 \mathrm{meV}$. This prediction is similar to the result reported in [13]. Furthermore, we find that the energy required for hydrogen release is $E_{r e l} \sim 550 \mathrm{meV}$.

The lower left panel of Fig. 1 shows the diffusion barriers for atomic hydrogen deep in the $\mathrm{Mg}$ bulk, again along the 'shaft' of fcc C-sites. The bond lengths of all atoms were allowed to relax in the dynamic case. We predict a very fast diffusion along this fcc 'shaft' (C-sites) with barriers $\Delta E_{b a r}=155 \mathrm{meV}$ and hence at room temperature $(T=300 \mathrm{~K})$ a predicted jump rate of

$$
\Gamma_{\text {bulk }}=\nu \exp \left(-\Delta E_{b a r} / k T\right)=2.4 \times 10^{10} \mathrm{~s}^{-1}
$$

where we have assumed a standard vibration prefactor $\nu=10^{13} \mathrm{~s}^{-1}$. However, we also predict, lower right panel, a large barrier $\Delta E_{\text {sbar }}=500 \mathrm{meV}$ to diffuse from the surface into the bulk. 
At room temperature the corresponding jump rate is $\Gamma_{\text {surface } \rightarrow \text { bulk }}=\nu \exp \left(-\Delta E_{\text {sbar }} / k T\right)=$ $4.0 \times 10^{4} \mathrm{~s}^{-1}$. While the $\mathrm{H}$ atoms can rapidly diffuse further into the bulk they must first overcome this significantly slower jump rate which constitutes a bottleneck. The predicted barrier for hydrogen entry limits the rate of hydrogen uptake and release and depreciates efficient storage operation. These bottleneck consequences motivate both a general focus on hydrogen-dynamics effects as well as specific efforts to lower the hydrogen-in-magnesium entry barrier by the use of rougher surfaces and formation of compound surface layers [12].

\section{Hydrogen storage in graphite}

The graphite supercell used in the calculations consists of two graphene sheets separated by $3.35 \AA$ and shifted relative to each other as shown in the inset of the right panel of Fig. 2. Each graphene sheet consists of eight carbon atoms with a nearest neighbor distance of $1.42 \AA$. The graphite supercell is large enough to avoid interactions between the hydrogen molecules in neighboring supercells. With the chosen size of the supercell the distance between neighboring $\mathrm{H}_{2}$ molecules is $4.9 \AA$ in the direction parallel to the sheets and $6.7 \AA$ in the perpendicular direction. The graphite-surface supercell is the same as the one described above, but with a vacuum region $13.69 \AA$ above the two graphene sheets. The bond length of the hydrogen molecule is held fixed at $0.76 \AA$.

We use both LDA and GGA for the exchange-correlation functional and a plane wave cutoff of 50 Ry (LDA) and 35 Ry (GGA).

In Fig. 2 we present the $\mathrm{H}_{2}$ adsorption energy as a function of distance. The left panel in Fig. 2 shows LDA and GGA results of the $\mathrm{H}_{2}$ adsorption on a graphite surface. We choose to present the results of the most attractive adsorption configuration [15, 9]. The adsorption distance is $2.7 \AA$ according to the LDA results and $3.5 \AA$ according to the GGA results. We also find significant differences between LDA and GGA in the binding energy at the adsorption position, 

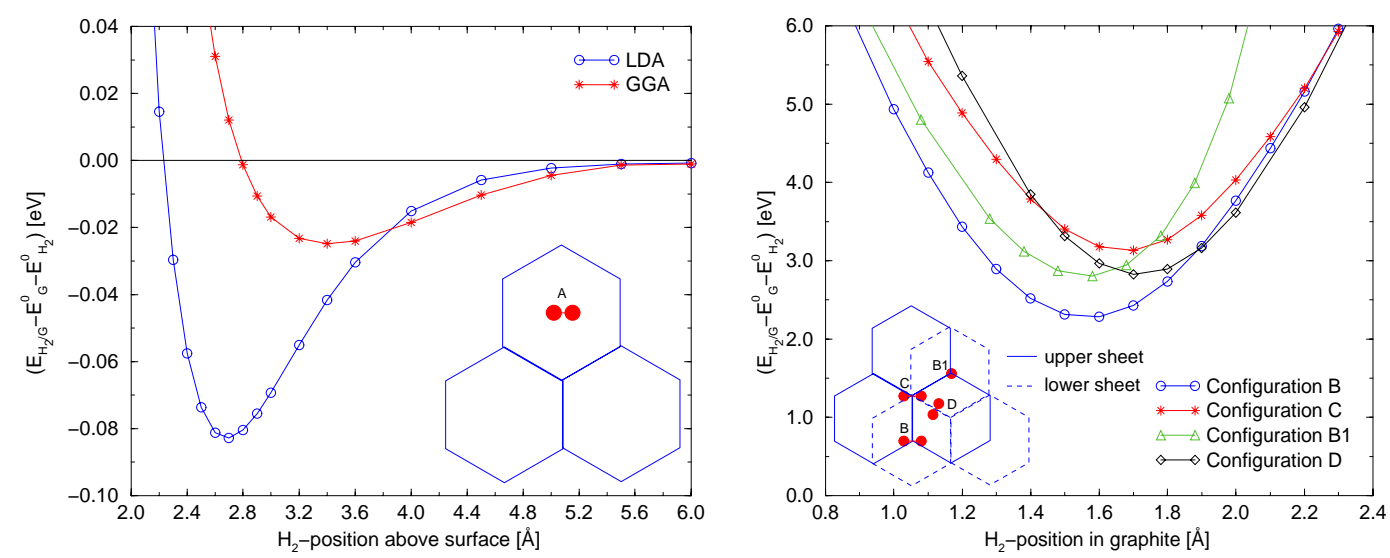

Figure 2: Adsorption of an $\mathrm{H}_{2}$ molecule on and inside graphite. The left panel compares LDA and GGA results for $\mathrm{H}_{2}$ adsorption on a graphite surface. While the results differ, both approximations predict a $\mathrm{H}_{2}$ /carbon separation comparable to the equilibrium interlayer separation $c_{0}=3.35 \AA$ in pure graphite. The right panel shows LDA results for adsorption of a single $\mathrm{H}_{2}$ molecule within bulk graphite. A single molecule can only cause local relaxation of the graphite. The molecule cannot easily fit between the graphite planes and requires large energies to be accommodated in any of the investigated configurations. The distance is measured from the $\mathrm{H}_{2}$ center of mass towards the lower graphite sheet; the $\mathrm{B}-\mathrm{D}$ configurations has the $\mathrm{H}_{2}$ axis parallel with the graphite planes while B1 is oriented perpendicular to these planes.

$83 \mathrm{meV}$ (LDA) and $25 \mathrm{meV}$ (GGA). The major differences between the LDA and GGA results show on problems in the description of the exchange and correlation effects.

For the investigation of graphite and graphite nanofibers as a potential storage system for hydrogen it is necessary to study the adsorption of $\mathrm{H}_{2}$ inside graphite. The results of the surface calculation, described above, show that the optimal $\mathrm{H}_{2}$-adsorption distance is far too large for energetically favorable adsorption of an $\mathrm{H}_{2}$ molecule between two graphene sheets inside graphite. This is visualized by the LDA results for several adsorption configurations in the right panel of Fig. 2. There is an energy cost of about $2.3 \mathrm{eV}$ for the most attractive configuration. $\mathrm{H}_{2}$ adsorption in graphite would be energetically more favorable with a larger 

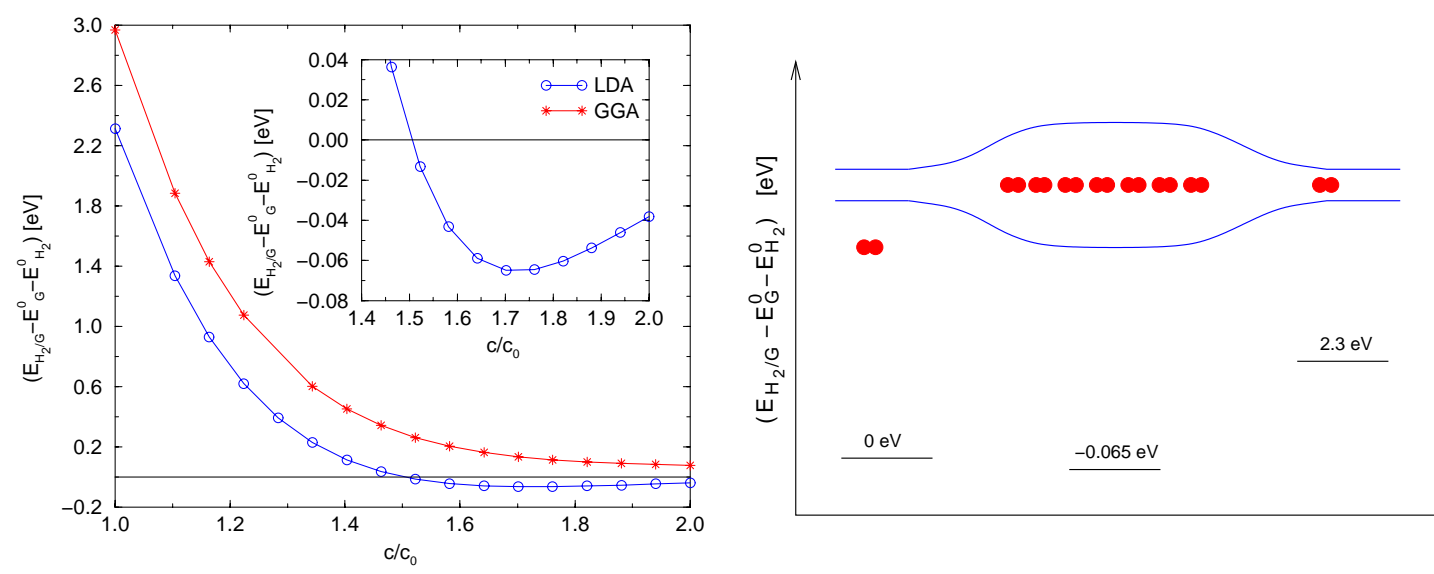

Figure 3: The left panel compares LDA and GGA results for graphite with full $\mathrm{H}_{2}$ coverage, i.e., with one $\mathrm{H}_{2}$ /hexagon. The inset is an enlargement of the LDA energy minimum that arises only after dramatic swelling of the interlayer separation of the graphite. The right panel shows schematic of the "self-sealing graphite containment" which results from this dramatic hydrogen-induced graphite swelling. The panel illustrates the change in the separation of the graphite layers and the corresponding variation in $\mathrm{H}_{2}$-in-graphite binding energies which results as a molecule moves from a dense storage pocket and out to a region of diluted $\mathrm{H}_{2}$ concentration en route to actual hydrogen release.

separation of the graphene sheets, and the above results can be regarded as the single- $\mathrm{H}_{2}$ adsorption limit, since one single $\mathrm{H}_{2}$ molecule will not be able to separate the graphene sheets. However, large amounts of $\mathrm{H}_{2}$ molecules between the sheets will cause separation.

To reach the goals set up by the U. S. Department of Energy it will be necessary to have equal amount of hydrogen and carbon atoms, which will result in a hydrogen content of $7.7 \mathrm{wt} \%$. This can, theoretically, be achieved with an $\mathrm{H}_{2}$ molecule on top of every hexagon in the graphene sheets. In the left panel of Fig. 3 we present results of LDA and GGA calculations with full coverage of hydrogen, i.e. $1 \mathrm{H}_{2}$ /hexagon $\left(\mathrm{H}_{2}\right.$ vertically oriented).

In these calculations we use a minimal supercell. We vary the distance between the graphene 
sheets, and at every distance we let the $\mathrm{H}_{2}$ molecules relax to reach the minimum energy of the system. The LDA results predict an optimum interlayer separation of 1.7 times that for pure graphite $\left(c=1.7 c_{0}\right)$, with a binding energy per $\mathrm{H}_{2}$ molecule of $65 \mathrm{meV}$. On the other hand, the GGA results do not show any energy minimum. We believe that the LDA results are more reasonable, and in this case graphite could be seen as a "self-sealing-container" for $\mathrm{H}_{2}$, as shown at the right in Fig. 3. The energy per $\mathrm{H}_{2}$ molecule at zero, single and full $\mathrm{H}_{2}$ coverage in graphite is shown in the right panel of Fig. 3 and it is seen to favor concentrated hydrogen distributions.

\section{Conclusions}

We find that both magnesium and graphite could be used for hydrogen storage. For magnesium, however, a significant barrier for hydrogen entry presents an important bottleneck. With one $\mathrm{H}_{2}$ molecule on top of every graphite hexagon, we find that hydrogen can be stored with an energy gain of $65 \mathrm{meV} / \mathrm{H}_{2}$ but with a dramatic swelling of the graphite interlayer separation.

\section{Acknowledgements}

This work was supported by the Swedish Research Council, the Carl Tryggers Stiftelse, and the Swedish Foundation for Strategic Research (SSF) through the consortium ATOMICS. 


\section{References:}

[1] M.S. Dresselhaus, K.A. Williams, and P.C. Eklund, MRS Bulletin/November 1999, 45.

[2] A.C. Dillon, K.M. Jones, T.A. Bekkedahl, C.H. Kiang, D.S. Bethune, and M.J. Heben, Nature (London) 386 (1997) 377.

[3] A. Chambers, C. Park, R.T.K. Baker, and N.M. Rodriguez, J. Phys. Chem. B 102 (1998) 4253.

[4] P. Chen, X. Wu, J. Lin, and L. Tan, Science 285 (1999) 91.

[5] R.T. Yang, Carbon 38 (2000) 623.

[6] M. Rzepka, P. Lamp, and M.A. de la Casa-Lillo, J. Phys. Chem. B 102 (1998) 10894.

[7] Q. Wang and J.K. Johnson, J. Chem. Phys. 110 (1999) 577.

[8] Q. Wang and J.K. Johnson, J. Phys. Chem. B 103 (1999) 4809.

[9] A.C. Johansson, L.H. Olesen, and T. Nielsen, Hydrogen storage in single walled carbon nanotubes, Dept. of Appl. Phys., Tech. Univ. of Denmark (2001).

[10] P. Hohenberg, W. Kohn, Phys. Rev. B 136 (1964) 864.

[11] W. Kohn and L. Sham, Phys. Rev. A 140 (1965) 1133.

[12] K.J. Gross, D. Chartouni, E. Leroy, A. Züttel, and L. Schlapbach, J. Alloys Comp. 269 (1998) 259.

[13] P.T. Sprunger and E.W. Plummer, Chem. Phys. Lett. 187 (1991) 559.

[14] D.M. Bird, L.J. Clarke, M.C. Payne, and I. Stich, Chem. Phys. Lett. 212 (1993) 518.

[15] J.S. Arellano, L.M. Molina, A. Rubio, and J.A. Alonso, J. Chem. Phys. 112 (2000) 8114. 